3. STELLAR ANGULAR DIAMETERS AND RADII 


\title{
STELLAR ANGULAR DIAMETER MEASUREMENTS BY INTERFEROMETRY
}

\author{
JOHN DAVIS \\ Chatterton Astronomy Department, School of Physics \\ University of Sydney, NSW 2006, Australia
}

\begin{abstract}
Stellar angular diameter measurements have been made with a range of interferometric techniques including speckle, aperture masking and long baseline optical/infrared interferometry. The current status of these measurements are summarised in terms of the range of spectral types and luminosity classes measured, the accuracies achieved, the wavelengths used for observations, and the reliability of the results. A number of major longbaseline interferometers are coming on-line, or are under development, and their potential is assessed in terms of wavelength cover, accuracy, angular resolution, and the range of spectral type and luminosity class cover.
\end{abstract}

\section{Introduction}

The angular diameters of stars are important for the determination of fundamental stellar properties including emergent fluxes, effective temperatures, radii and absolute luminosities, and for providing constraints on theoretical stellar models. In this contribution the emphasis is on their determination by means of interferometry.

The first angular diameter of a star ( $\alpha$ Ori) was measured interferometrically by Michelson and Pease in 1921. Although they succeeded in measuring 6 late type giants and supergiants, the accuracy was poor, and the technique was limited by the technology available and the deleterious effects of atmospheric turbulence. The field did not progress significantly until Hanbury Brown and Twiss developed the technique of intensity interferometry in the 1950s. This overcame the problems posed by atmospheric turbulence and led to the Narrabri Stellar Intensity Interferometer (NSII). Since this meeting marks Hanbury Brown's 80th birthday, a brief list of the achievements made with the NSII under his leadership is included:

T.R. Bedding et al. (eds.),

Fundamental Stellar Properties: The Interaction between Observation and Theory, 31-38.

(C) 1997 IAU. Printed in the Netherlands. 
- Angular diameters for 32 early type stars, including main-sequence stars, which led to an effective temperature scale for stars hotter than the Sun. These are still the only measurements of early-type stars.

- The distance, the mass, radius and luminosity of the primary, and the mass of the secondary for a double-lined spectroscopic binary ( $\alpha$ Vir).

- Performance of a number of exploratory experiments including:

- Measurement of the angular size of the emission envelope surrounding a Wolf-Rayet star $\left(\gamma^{2}\right.$ Vel $)$.

- An attempt to measure limb-darkening ( $\alpha \mathrm{CMa})$.

- Measurement of an extended corona ( $\beta$ Ori).

- An attempt to measure the distortion of a rapidly rotating star $(\alpha \mathrm{Aql})$.

Although the intensity interferometer has now been superseded, because of its inherent low sensitivity, by various forms of amplitude interferometer, the NSII demonstrated that angular diameters of stars can be determined accurately through the earth's atmosphere.

As the achievements of the NSII illustrate, interferometry can do a great deal more than measure the angular diameters of single stars, but this will be the subject of other contributions to this meeting.

\section{Techniques and Instruments}

Several interferometric techniques have been used for the determination of stellar angular diameters and, very briefly, they are:

- Speckle - limited by the diameter of the largest telescope apertures to resolve only a small number of stars for angular diameter measurements.

- Aperture Masking (Non-Redundant Masking or NRM) - has some advantages over speckle but is also limited in resolution by telescope aperture diameters. Both speckle and NRM complement long-baseline interferometers by providing observations at the short baselines generally inaccessible to multi-aperture instruments.

- Intensity - overcomes the effects of atmospheric turbulence but limited in sensitivity. The only instrument (now closed) was the NSII.

- Amplitude (Modern Michelson) - the very high angular resolution, longbaseline optical/infrared instruments of today are of this type.

Table 1 lists the amplitude interferometers which have been developed, are under construction, or are being planned. These are all ground-based instruments. Space based interferometer projects are under development but generally with objectives other than stellar angular diameter determinations. 
TABLE 1. Long-baseline optical/infrared amplitude interferometers

\begin{tabular}{lllrcl}
\hline Instrument & Location & $\begin{array}{c}\text { Aperture } \\
\text { Diameter } \\
(\mathrm{m})\end{array}$ & $\begin{array}{c}\text { Max. } \\
\text { B'line } \\
(\mathrm{m})\end{array}$ & $\begin{array}{c}\lambda \\
\text { Range } \\
(\mu \mathrm{m})\end{array}$ & Status \\
\hline SUSI Prototype & Australia & $2 \times 0.10$ & $\sim 13$ & $0.4-0.5$ & Closed \\
Mark III & USA & $2 \times 0.05$ & $\sim 32$ & $\begin{array}{l}0.45-0.8 \\
\text { Closed }\end{array}$ & $\begin{array}{l}\text { Visible } \\
\text { I2T }\end{array}$ \\
FI2T & France & $2 \times 0.26$ & 144 & Working \\
SUSI & Australia & $2 \times 0.14$ & 640 & $0.4-0.9$ & Working to 80 m \\
IOTA & USA & $3 \times 0.45$ & 38 & Vis/IR & 2 apert. working \\
COAST & UK & $4 \times 0.4$ & 100 & Red/IR & 3 apert. working \\
Palomar Interf. & USA & $2 \times 0.4$ & 100 & 2.2 & Commissioning \\
NPOI (Astrom.) & USA & $4 \times 0.125$ & 38 & $0.45-0.9$ & Commissioning \\
NPOI (Imaging) & USA & $6 \times 0.35$ & 437 & $0.45-0.9$ & Construction \\
CHARA Array & USA & $7 \times 1$ & 354 & $0.55-0.9$ & Construction \\
& & & & $2.1-2.5$ & \\
VLTI & Chile & $4 \times 8$ plus & 200 & $0.45-20$ & Construction \\
& & $3 \times 1.8$ & & & Finalising design \\
Keck & USA & $2 \times 10$ plus & 165 & $2.2-10$ & \\
& & SideKecks & & & Planned \\
\hline
\end{tabular}

\section{The Measurement of Angular Diameters by Interferometry}

In principle, the response of an interferometer to a star, as a function of baseline, is the Fourier transform of the brightness distribution across the equivalent strip source. It is not appropriate to go into details but there are certain aspects important in the context of angular diameter determinations. If both the phase and visibility of the interference fringes could be measured, a unique solution for the brightness distribution could be determined which would include asymmetries due to spots etc. In practice, for measures made with a single baseline at a time, which has generally been the case, only the fringe visibility is meaningful and symmetry in the brightness distribution has to be assumed. In most cases this is a reasonable assumption.

Assuming symmetry in the brightness distribution, the response of an interferometer depends on the angular size and the limb-darkening of the stellar disk. In principle, from measurements of fringe visibility as a function of baseline, one should be able to determine the limb-darkened angular diameter of the star. Unfortunately this turns out to be extremely difficult. The effect of limb darkening on the shape of the response of an interferometer is very small-too small to measure with the accuracy required to 
distinguish between different limb-darkening laws (Hanbury Brown et al., 1974b). The main difference is in the scale of the responses which varies from 1.0 for a uniformly bright disk to $\sim 1.13$ for a fully darkened disk. Unfortunately, this cannot be determined but it has been shown, both from accurate measurements of visibility around the first zero in the response and from the variation of angular diameter with wavelength, that observations are consistent with the predictions of model atmospheres.

The general practice has been to fit the response for a uniform disk to determine an angular diameter and then to convert it, using data from atmospheric models, to obtain an estimate of the limb-darkened angular diameter. The magnitude of the correction is up to $\sim 10 \%$ with an uncertainty of the order of $10 \%$. The result is a systematic uncertainty in the limbdarkened angular diameter of the order of $1 \%$ in addition to the measurement uncertainty of the uniform disk angular diameter. This must be borne in mind in any discussion of the final accuracies of stellar angular diameters.

In summary, ideally the limb-darkened diameter would be measured but the vast majority of angular diameter measurements in the literature have been determined as uniform disk diameters and subsequently converted to limb-darkened diameters.

\section{Stellar Angular Diameters}

\subsection{THE DATA}

A database of 490 interferometric measurements of the angular diameters of 156 stars has been assembled. These data have been obtained using speckle, NRM, intensity and amplitude interferometry and much of the data have been taken from the literature (space limitations do not permit a listing of the sources). I am grateful to David Mozurkewich for allowing me to include unpublished results for 78 stars obtained with the Mark III interferometer. More than $98 \%$ of the angular diameters are uniform disk determinations.

Figure 1(a) shows, in a fairly coarse grid, the distribution of 145 measured stars as a function of spectral type and luminosity class. All stars, irrespective of the accuracy of the measured angular diameter have been included. The entries for the early-type stars (O and $\mathrm{B}$ ) are all from the NSII as are most of the A-type stars. There are no measurements for mainsequence stars later than A7. In Fig. 1(b) the entries have been restricted to measurements with an uncertainty $\leq 5 \%$. The range of spectral types and luminosity classes covered is poorer with only late-type giants and supergiants well represented. This trend is emphasised if entries are restricted to measurements with smaller uncertainties.

The major bodies of data come from the NSII and Mark III instruments. As a first step in examining the accuracies of angular diameter measure- 


\begin{tabular}{|c|c|c|c|c|c|}
\hline \multirow{2}{*}{$\begin{array}{c}\text { Range in } \\
\text { Spectral } \\
\text { Type }\end{array}$} & \multicolumn{5}{|c|}{ Luminosity Class } \\
\hline & 1 & II & III & IV & $\mathrm{V}$ \\
\hline 0 & 3 & 濨 & TOM & The & 1 \\
\hline B0-B4 & 2 & 2 & 3 & 2 & 2 \\
\hline B5-B8 & 2 & $\mathbb{Z}_{x}$ & 1 & 1 & 1 \\
\hline $\mathrm{AO}-\mathrm{A} 3$ & 1 & 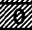 & Thes & 2 & 5 \\
\hline A5-A7 & 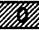 & 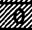 & 1 & T/U & 1 \\
\hline FO-F5 & 3 & 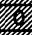 & 78 & 1 & Ty \\
\hline F8 & 2 & 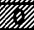 & W & Th & -3 \\
\hline G0-G5 & 2 & 1 & 2 & 3 & 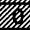 \\
\hline G7-G9.5 & 2 & 1 & 12 & 10 & m \\
\hline KO-K3.5 & 3 & 10 & 17 & 6 & $x$ \\
\hline K4-K7 & 1 & 1 & 7 & 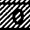 & 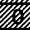 \\
\hline M0-M4 & 5 & 6 & 18 & 6 & 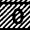 \\
\hline M5-M8 & 1 & 2 & 15 & 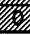 & 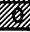 \\
\hline TOTAL: & 27 & 23 & 76 & 9 & 10 \\
\hline
\end{tabular}

(a)

\begin{tabular}{|c|c|c|c|c|c|}
\hline \multirow{2}{*}{$\begin{array}{c}\text { Range in } \\
\text { Spectral } \\
\text { Type }\end{array}$} & \multicolumn{5}{|c|}{ Luminosity Class } \\
\hline & 1 & II & III & IV & $\mathrm{V}$ \\
\hline $\mathrm{O}$ & Ty & Yut & 兓 & Tys & Ty \\
\hline B0-B4 & M & 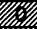 & 1 & 1 & 1 \\
\hline B5-B8 & 1 & 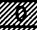 & 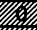 & Ty & 1 \\
\hline$A 0-A 3$ & 1 & 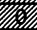 & 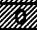 & 1 & 3 \\
\hline A5-A7 & 㦈 & 埩 & y & The & 1 \\
\hline F0-F5 & 2 & 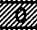 & - & 1 & 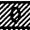 \\
\hline F8 & 2 & 列 & H & 18 & 3 \\
\hline G0-G5 & 2 & 1 & 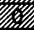 & 3 & 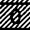 \\
\hline G7-G9.5 & 2 & 1 & 11 & ty & 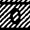 \\
\hline K0-K3.5 & 3 & 7 & 11 & 3 & 3 \\
\hline K4-K7 & 1 & T现 & 6 & v & o \\
\hline MO-M4 & 5 & 6 & 15 & S & 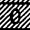 \\
\hline M5-M8 & 1 & 㱛 & 9 & - & 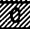 \\
\hline TOTAL: & 20 & 15 & 53 & 6 & 6 \\
\hline
\end{tabular}

(b)

Figure 1. The distribution of interferometric angular diameter measurements as a function of spectral type and luminsity class: (a) all measurements regardless of accuracy; (b) measurements with an uncertainty in angular diameter $\leq \pm 5 \%$.

ments, the uncertainties for these two groups of data are plotted against visual magnitude in Figs. 2 and 3.

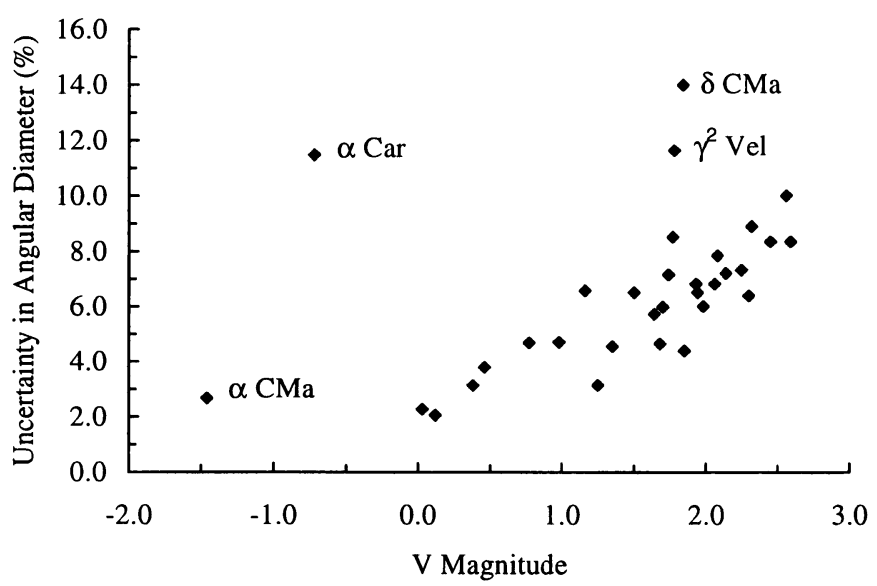

Figure 2. The percentage uncertainty in angular diameter measurements for 32 stars measured at $\lambda=443 \mathrm{~nm}$ with the Narrabri Stellar Intensity Interferometer as a function of visual magnitude.

For the NSII data the uncertainties range from $\sim 2 \%$ at $\mathrm{V}=0$ to $\sim 10 \%$ at $\mathrm{V}=2.5$. There are four stars which lie off the main trend. The plotted uncertainty for $\alpha \mathrm{CMa}$ is the published value (Hanbury Brown et al., 1974a) which was arbitrarily double the formal uncertainty because it was felt at the time that unidentified systematic errors of the order of $\sim 1 \%$ might be 
present. Subsequent measurements with the SUSI prototype and with SUSI agree with the NSII value within the formal error so the uncertainty plotted in Fig: 2 should be halved. $\alpha$ Car was measured low down on the visibility curve resulting in low accuracy, $\gamma^{2}$ Vel is a binary and $\delta$ CMa was the faintest star at $\lambda 443 \mathrm{~nm}$ measured with the NSII.

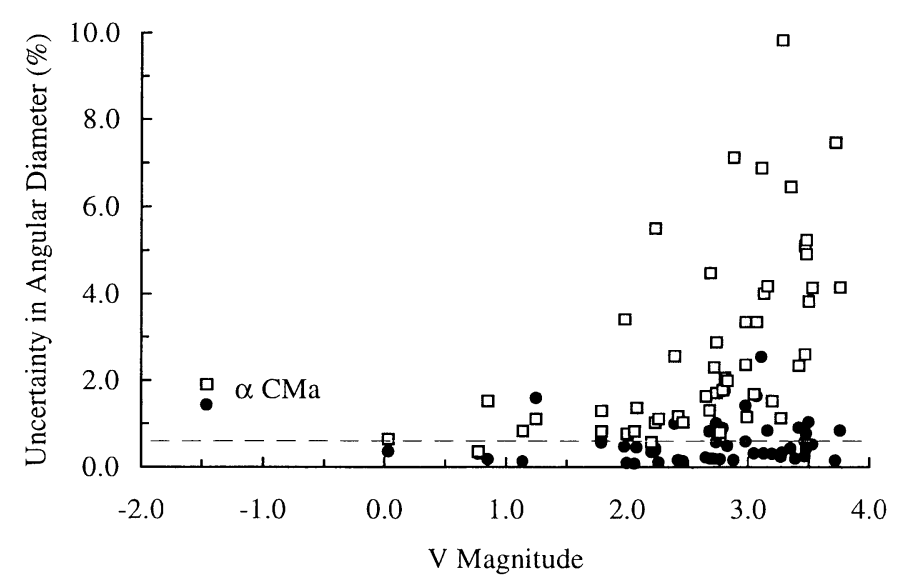

Figure 3. The percentage uncertainty in angular diameter measurements measured with the Mark III interferometer as a function of visual magnitude. Key: $\square$ measurements made at $\lambda=451 \mathrm{~nm} ; \bullet$ measurements made at $\lambda=800 \mathrm{~nm}$.

In the case of the Mark III data, measurements were made at four different wavelengths but not for all stars. In Fig. 3 the data for the longest $(800 \mathrm{~nm})$ and shortest $(451 \mathrm{~nm})$ wavelengths are plotted. The results for $800 \mathrm{~nm}$ have smaller errors reflecting the fact that the deleterious effects of atmospheric turbulence decrease with increasing wavelength. In many cases the formal errors are a small fraction of $1 \%$ but Mozurkewich (1997) considers that the real uncertainty is not less than $\sim 0.5 \%$ due to residual systematic errors and may be of the order of $1 \%$. However, the plot shows that it is possible to reduce the measurement errors to at least the $1 \%$ level even in the blue. This accords with experience obtained with SUSI in the blue $(\lambda 442 \mathrm{~nm})-$ although there is not yet a large body of data, calibration procedures have been developed that give angular diameters to better than $2 \%$ and in some cases $\leq 1 \%$. Angular diameters with uncertainties $\leq 1 \%$ have also been obtained with the I2T and IOTA instruments at $\lambda 2.2 \mu \mathrm{m}$.

\subsection{THE RELIABILITY OF ANGULAR DIAMETER MEASUREMENTS}

Formal uncertainties and estimates of systematic errors are indicators of reliability but the real test is how well measurements made with different instruments and techniques agree. With this in mind, stars in the database 
measured by more than one instrument or technique have been identified. In addition, stars in the interferometric database that have lunar occultation angular diameter measurements listed in the catalog published by White and Feierman (1987) have also been identified. The resulting list of stars with more than one angular diameter determination was analysed as follows:

1. Measurements with uncertainties $>10 \%$ were removed.

2. Formal uncertainties less than $0.5 \%$ for the Mark III data were increased to $0.5 \%$. This is the best estimate of the uncertainty when systematics are taken into account (Mozurkewich, 1997).

3. In each comparison only measurements at the same wavelength or within $50 \mathrm{~nm}$ of each other were retained in order to avoid significant wavelength effects. TiO band measurements were omitted.

4. For each remaining star with 2 or more measurements a weighted mean for the angular diameter was calculated.

5. The difference between each measurement and its associated weighted mean was expressed in terms of the measurement's uncertainty.

6. The results are plotted in Fig. 4.

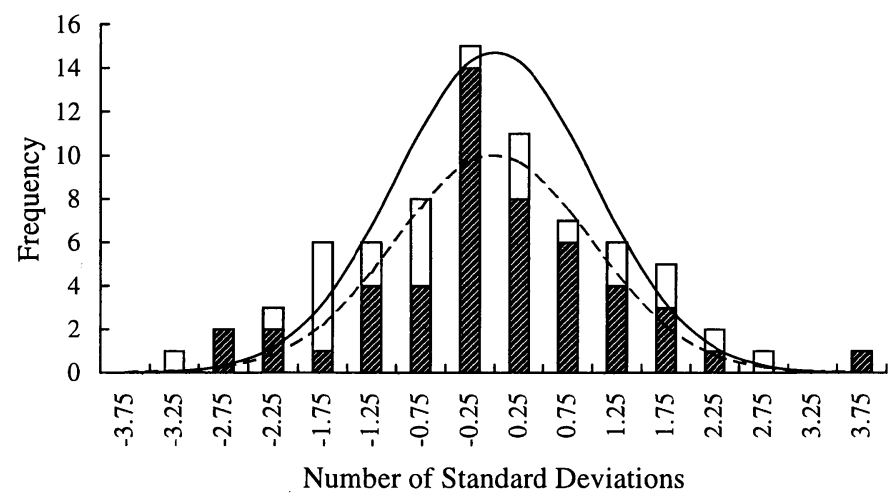

Figure 4. The distribution of angular diameters determined by different instruments or techniques expressed in terms of their standard deviations from the weighted mean angular diameter for each star. The hatched areas are for stars observed by two or more interferometric groups and the clear areas for stars observed by lunar occultation and interferometry. The normal error curves have been fitted to the data: the full curve is for the entire data set and the dashed curve for the interferometric data alone.

The distribution for interferometric measurements alone in Fig. 4 is in reasonable agreement with the fitted normal error curve indicating that there are no major systematic differences between the results for different instruments and techniques. The agreement in the case of interferometric and lunar occultation measurements with the normal error curve is not so good, sug- 
gesting that there may be some systematic effects in the occultation data which have not been included in the assessment of the uncertainties.

\section{The Prospects}

There are several high angular resolution interferometers coming into operation, under construction, or being planned. These are summarised in Table 1 . The wavelengths covered range from $\sim 440 \mathrm{~nm}$ to $20 \mu \mathrm{m}$ and planned baselines range up to $640 \mathrm{~m}$. It should be noted that a combination of the longest baselines and shortest wavelengths will be necessary to measure a sample of the hottest stars. Conversely, larger apertures operating in the red or near infrared will be required to measure stars at the cool end of the main sequence. Referring to Fig. 1, we can expect measurements of essentially all spectral types and classes with the instruments listed in Table 1 except for the coolest main-sequence stars but, even here, it should be possible to fill in down to at least the M6 dwarfs with, for example, the VLTI.

Current data suggest that formal fitting errors can be reduced to $<1 \%$ in the blue and $<0.5 \%$ at red wavelengths and that systematic errors can be reduced to this level. Nevertheless, it is highly desirable that the new instruments observe common stars to establish the reliability of the measurements more rigorously than has been possible with existing data.

In discussing the accuracies of angular diameter determinations it must be remembered that equivalent uniform disk diameters determined from observational data have to be scaled using an assumed limb-darkening law to obtain true disk diameters. The current uncertainty in the scaling, which is a systematic error, is comparable with or greater than the fitting uncertainty. An investigation into the significance of accurate measurements at multiple wavelengths for providing constraints on limb-darkening laws should be undertaken. It is also important that the observational data (visibility values v. baseline) are published with the equivalent uniform disk diameters. For large bodies of data this may not be feasible but they should be made available so that, as our knowledge of limb darkening improves, it will be possible for limb-darkened models to be fitted directly to the observational data.

\section{References}

Hanbury Brown, R., Davis, J. and Allen, L.R. (1974a) The angular diameters of 32 stars, Mon. Not. R. Astron. Soc., 167, pp. 121-136

Hanbury Brown, R., Davis, J. and Allen, L.R. (1974b) The effects of limb darkening on measurements of angular size with an intensity interferometer, Mon. Not. R. Astron. Soc., 167 , pp. $475-484$

Mozurkewich, D. (1997) Private communication

White, N.M. and Feierman, B.H. (1987) A catalog of stellar angular diameters measured by lunar occultations, Astron. J., 94, pp. 751-770 